\title{
9
}

\section{LAS ASAMBLEAS ANUALES DEL FMI DE OCTUBRE DE 2019}

Las pasadas asambleas anuales del FMI, de octubre de 2019, han sido las primeras bajo el mandato recién estrenado como directora gerente de Kristalina Georgieva y se han desarrollado bajo un contexto de tensiones geopolíticas (brexit, guerra comercial entre EE UU y China) frente a las que Georgieva ha respondido con un llamamiento a superar las diferencias mediante cooperación multilateral. Por otro lado, la recta final previa ha venido marcada por el hecho de que estas asambleas habían sido señaladas como plazo límite para alcanzar un acuerdo sobre la renovación de recursos del FMI (Decimoquinta Revisión General de Cuotas), que finalmente ha sido necesario postergar. Como de costumbre, en paralelo a las asambleas se ha celebrado una reunión de ministros de Finanzas y gobernadores de bancos centrales del G20, cuyos principales resultados han sido la publicación de sendos comunicados sobre fiscalidad internacional y las mal denominadas global stablecoins, y en la que se han perfilado las prioridades de la presidencia saudí del G20 en 2020.

Palabras clave: FMI, cuotas, G20, cooperación multilateral, previsiones de crecimiento.

Clasificación JEL: F00, F02.

\section{Introducción}

Las recientes asambleas anuales del FMI, de octubre de 2019, se han celebrado en un contexto marcado por tres peculiaridades: primero, la persistencia de tensiones geopolíticas con implicaciones económicas directas

* Secretaría General del Tesoro y Financiación Internacional, Ministerio de Economía y Empresa. Este artículo ha sido elaborado por Ana Fornells de Frutos, María Mateo Feito y Juan Luis Díez Gibson.

Versión de diciembre de 2019.

DOI: https://doi.org/10.32796/bice.2020.3119.6959 (señaladamente, el brexit y la guerra comercial entre EE UU y China), lo que ha marcado las discusiones sobre la situación de la economía global y las consiguientes recomendaciones de política económica; segundo, estas asambleas marcaban, en principio, el plazo máximo para alcanzar un acuerdo sobre los recursos del FMI bajo la denominada Decimoquinta Revisión General de Cuotas, que finalmente se ha tenido que postergar; tercero, han sido las primeras asambleas bajo el mandato de Kristalina Georgieva como nueva directora gerente, lo que ha generado una expectación adicional. 


\section{Perspectivas de la economía mundial}

\subsection{Situación macroeconómica}

Las asambleas anuales de octubre de 2019 se han celebrado en un contexto de desaceleración macroeconómica mundial. Así, y tal y como se aprecia en la Tabla 1, el FMI prevé una caída del crecimiento de la economía mundial desde un 3,6\% en 2018 hasta un $3 \%$ en 2019, lo que supone la tasa más baja desde el estallido de la crisis financiera global en 20082009. Aunque para 2020 se prevé un repunte del crecimiento respecto a 2019 , hasta un $3,4 \%$ -que se estima se mantendrá hasta 2024-, este se sustenta en el mayor dinamismo de unas economías emergentes que muestran, en general, una situación frágil.
Por otro lado, el FMI ha revisado a la baja sus previsiones de crecimiento del PIB para la economía mundial, tanto en 2019 como en 2020, debido al empeoramiento de las perspectivas en las economías tanto avanzadas como emergentes.

Tanto la desaceleración como la revisión a la baja de las perspectivas de crecimiento se deben al empeoramiento de la situación en la industria manufacturera y al impacto negativo de las tensiones comerciales sobre la inversión, factores negativos que han tenido mayor peso que la evolución positiva en términos de dinamismo en la creación de empleo y el sector servicios. Por otro lado, este empeoramiento de la situación de la economía global se produce a pesar de la amplia adopción de medidas fiscales y monetarias de estímulo.

TABLA 1

PREVISIONES DEL FMI SOBRE CRECIMIENTO DEL PIB

\begin{tabular}{|c|c|c|c|c|c|}
\hline & \multirow[t]{2}{*}{2018} & \multicolumn{2}{|c|}{$\begin{array}{l}\text { Previsiones de } \\
\text { octubre de } 2019\end{array}$} & \multicolumn{2}{|c|}{$\begin{array}{c}\text { Diferencias respecto a la } \\
\text { actualización de julio de } 2019\end{array}$} \\
\hline & & 2019 & 2020 & 2019 & 2020 \\
\hline Output mundial & 3,6 & 3,0 & 3,4 & $-0,2$ & $-0,1$ \\
\hline Economías avanzadas & 2,3 & 1,7 & 1,7 & $-0,2$ & 0,0 \\
\hline Estados Unidos & 2,9 & 2,4 & 2,1 & $-0,2$ & 0,2 \\
\hline Zona euro & 1,9 & 1,2 & 1,4 & $-0,1$ & $-0,2$ \\
\hline Alemania & 1,5 & 0,6 & 1,4 & $-0,1$ & $-0,3$ \\
\hline Francia & 1,7 & 1,2 & 1,3 & $-0,1$ & $-0,1$ \\
\hline Italia & 0,9 & 0,0 & 0,6 & $-0,1$ & $-0,2$ \\
\hline España & 2,6 & 2,2 & 1,8 & $-0,1$ & $-0,1$ \\
\hline Reino Unido & 1,4 & 1,2 & 1,5 & $-0,1$ & 0,1 \\
\hline Japón & 0,8 & 0,9 & 0,5 & 0,0 & 0,1 \\
\hline Canadá & 1,9 & 1,5 & 1,8 & 0,0 & $-0,1$ \\
\hline Economías emergentes y en desarrollo & 4,5 & 3,9 & 4,5 & $-0,2$ & $-0,2$ \\
\hline Rusia & 2,3 & 1,1 & 1,9 & $-0,1$ & 0,0 \\
\hline China & 6,6 & 6,1 & 5,8 & $-0,1$ & $-0,2$ \\
\hline India & 6,8 & 6,1 & 6,8 & $-0,9$ & $-0,4$ \\
\hline Brasil & 1,1 & 0,9 & 2,0 & 0,1 & $-0,4$ \\
\hline México & 2,0 & 0,4 & 1,5 & $-0,5$ & $-0,4$ \\
\hline Comercio mundial (volumen) & 3,5 & 1,2 & 3,2 & $-1,3$ & $-0,5$ \\
\hline Precios del petróleo & 29,4 & $-11,4$ & $-8,3$ & $-7,3$ & $-5,8$ \\
\hline
\end{tabular}


Respecto a las economías avanzadas, la ralentización en el crecimiento en 2019, respecto a los niveles de 2017-2018 en la zona euro, América del Norte y las economías desarrolladas asiáticas, se ha debido, en gran medida, a la importante desaceleración en la producción industrial, consecuencia asimismo de: (i) una menor demanda externa (incluyendo la procedente de China); (ii) las repercusiones de las tensiones comerciales y del incremento de la incertidumbre, que han reducido el comercio y las inversiones; y (iii) una reducción muy significativa en la producción global de automóviles (especialmente notable en países como Alemania). A medio plazo, el FMI estima que el crecimiento de la actividad en las economías avanzadas se mantendrá a niveles modestos, como consecuencia de un incremento moderado de la productividad y del envejecimiento de la población, que limitará la población activa.

En cuanto a los principales riesgos a la baja para la economía mundial, el FMI destaca cuatro:

- Que no se cumplan las previsiones de mejora de la situación de aquellas economías emergentes que se han visto afectadas por episodios de estrés severo en 2019 o/y de aquellas cuyas economías han crecido por debajo de su media histórica.

- Que se produzca una escalada en las tensiones comerciales, lo que generaría un incremento en la incertidumbre global. A medio plazo, además, podría generarse una ruptura de las cadenas de suministro globales, que amplificaría la crisis en el sector industrial y que podría transmitirse también a los mercados financieros.

- Que se produzcan episodios de crisis en algunos mercados financieros, como consecuencia de un deterioro en las expectativas, lo que podría derivar en un endurecimiento de las condiciones financieras a las que se enfrentan los países más vulnerables. Estos episodios de crisis podrían desencadenarse, por ejemplo, por un empeoramiento sustancial en las tensiones comerciales y/o geopolíticas, por una salida sin acuerdo de la UE por parte del Reino Unido (brexit duro) o por una sucesión de malos datos económicos que se interpreten como anticipo de un parón en el crecimiento.

- Que, a medio plazo, la falta de actuación a la hora de combatir el cambio climático haga que se deterioren las expectativas de crecimiento de aquellas economías vulnerables más expuestas a los efectos negativos del mismo.

\subsection{Situación financiera}

Respecto a las vulnerabilidades financieras, el FMI destaca los desequilibrios provenientes del periodo prolongado de tipos mínimos (lower for longer), el cual ha conducido a una relajación de las condiciones de financiación en las economías avanzadas y a una intensificación de la búsqueda de rentabilidad (search for yield) por parte de los inversores. Ello se produce, además, en un contexto de incremento de la deuda corporativa, una mayor presencia de activos poco líquidos en los balances de los fondos de inversión y un incremento de la deuda externa en las economías emergentes.

\subsection{Recomendaciones}

A nivel multilateral, el FMI defiende dos líneas de actuación principales: por un lado, $\triangleright$ 
llegar a acuerdos internacionales para resolver los conflictos comerciales y eliminar las distorsiones generadas por las barreras comerciales impuestas recientemente; por otro lado, reducir las emisiones de efecto invernadero para mitigar el incremento global en las temperaturas y las catástrofes derivadas del cambio climático.

A nivel nacional, el FMI defiende tres políticas dirigidas a estabilizar el nivel de actividad y fortalecer las bases que permitan un crecimiento sostenido: primero, mantener una política monetaria acomodaticia, aunque permaneciendo alertas sobre la necesidad de adoptar medidas de supervisión macroprudencial y un enfoque proactivo en la supervisión para evitar el incremento en las vulnerabilidades que puede originar una política monetaria laxa; segundo, un papel más activo de la política fiscal, que ha de ser expansiva en aquellos países con margen fiscal, mientras que ha de ralentizarse el ritmo de consolidación en aquellos países sin el mismo; tercero, aprovechar la oportunidad que brinda la situación actual de tipos de interés muy bajos para liberar recursos que, de otra manera, tendrían que dedicarse al pago de intereses, siempre que no haya riesgo para la sostenibilidad de la deuda y priorizando medidas encaminadas a incrementar la capacidad productiva, tales como la inversión en infraestructuras para combatir el cambio climático.

\section{Decimoquinta Revisión General de Cuotas}

El FMI es un fondo común de divisas de los 189 países miembros, que se aportan en forma de cuotas, cuyo volumen global y asignación por países se revisa cada cinco años mediante un proceso políticamente difícil y sujeto a retrasos. Por ello, en momentos de crisis financiera o elevados riesgos globales, las cuotas se han suplementado con los llamados «recursos prestados» (préstamos multilalerales - new arrangements to borrow, NAB-y préstamos bilaterales), que se entienden como líneas de defensa adicionales ante posibles necesidades sobrevenidas de financiación.

A día de hoy, el fondo cuenta con 959.000 millones de derechos especiales de giro (en torno a 1,2 billones de euros) en recursos, entre cuotas y recursos prestados, lo cual supone un volumen de recursos tres veces superior al existente en 2008. Teniendo en cuenta las reservas, límites de préstamo y requisitos de activación de las diferentes líneas de defensa, en la práctica esto se traduce en una capacidad de préstamo de 693.000 millones de derechos especiales de giro (en torno a 0,86 billones de euros). Aproximadamente, la mitad son cuotas y la otra mitad, recursos prestados.

Dado que entre 2020 y 2022 vencen cerca de la mitad de los recursos prestados de que dispone el fondo, cobra especial relevancia la Decimoquinta Revisión General de Cuotas, actualmente en curso, para tratar de evitar una merma en la capacidad potencial de actuación de la institución ante una situación de crisis. Aunque estaba previsto que esta revisión se completara, como muy tarde, en estas últimas reuniones anuales de octubre de 2019, la falta de un pronunciamiento claro al respecto por parte de EE UU ha obligado finalmente a posponer la decisión.

El escenario base de acuerdo sobre la ampliación de recursos consiste actualmente en una duplicación de los NAB y el cierre de una ronda adicional de préstamos bilaterales. Con ello, se permitiría mantener el volumen actual de recursos del FMI. Como referencia respecto a la posición de EE UU, hay que tener en $\triangleright$ 


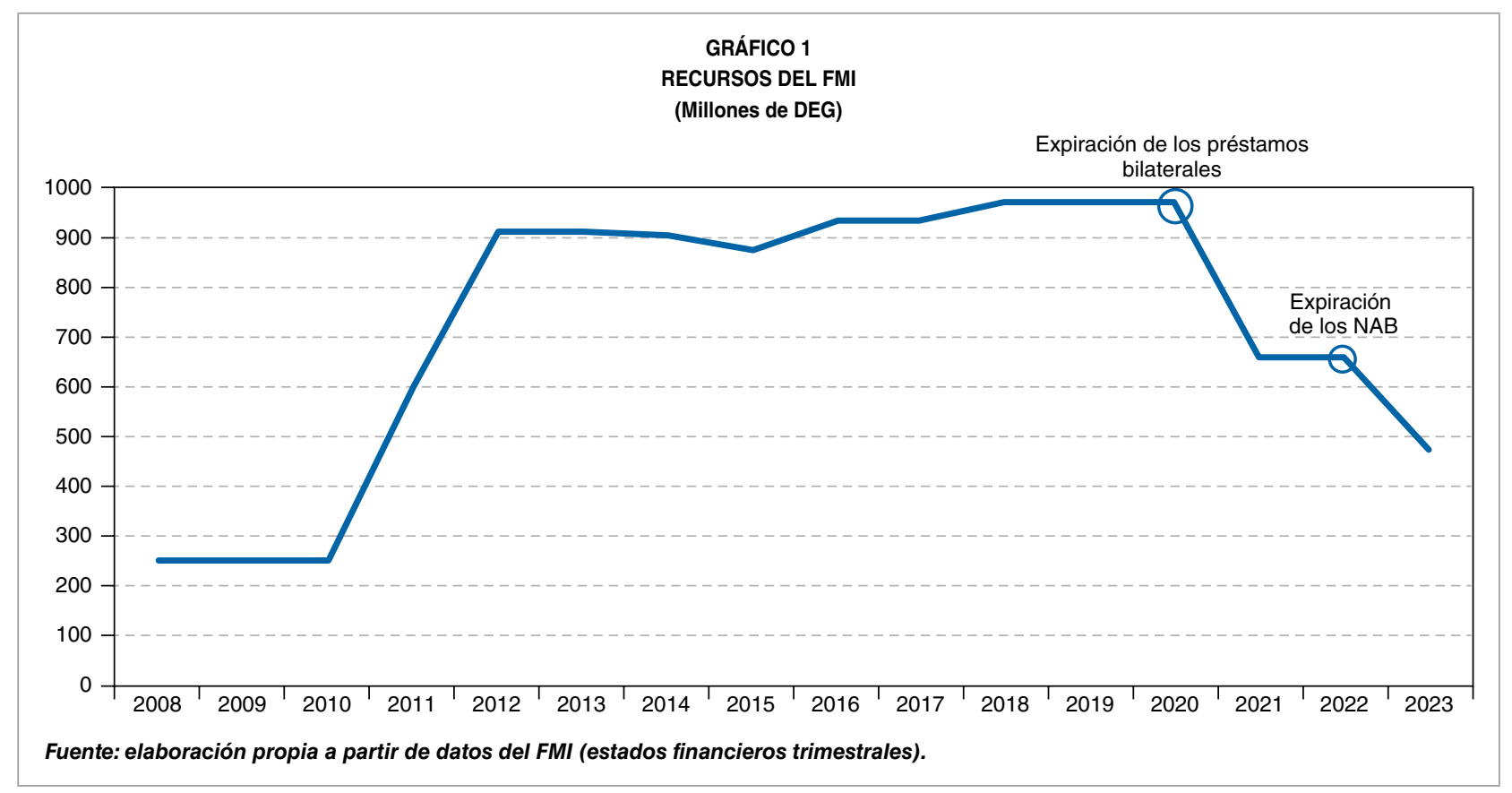

cuenta la declaración pública de David Malpass, exsubsecretario del Tesoro, en el Senado estadounidense el pasado 27 de noviembre de 2018, en la que vinculaba de algún modo la Decimoquinta Revisión General de Cuotas del FMI a las revisiones en curso de las remuneraciones del personal del FMI (para hacerlas menos costosas y más eficientes) y la condicionalidad en los programas de préstamos del FMI (para orientarla en mayor medida hacia el crecimiento).

\section{Prioridades y retos}

A iniciativa propia, desde 2012, Christine Lagarde comenzó a publicar de forma semestral, con ocasión de las asambleas de abril y octubre, su Agenda Mundial de Políticas (Global Policy Agenda) como directora gerente. Por primera vez, bajo el mando de Kristalina Georgieva, aunque siguiendo el enfoque de los dos últimos años, el de la agenda de estas últimas asambleas ha estado centrado en la necesidad de cooperación global (superar las diferencias mediante la cooperación multilateral), especialmente para encontrar soluciones duraderas a las disputas comerciales, pero también para llegar a un acuerdo sobre la fiscalidad digital y preservar las reformas en la regulación financiera internacional adoptadas tras la crisis, así como evitar una acumulación excesiva de deuda en países en desarrollo. Adicionalmente, se pone el foco en retos de largo plazo como la creciente desigualdad y los retos demográficos.

\section{Reunión ministerial del G20: fiscalidad internacional y las mal denominadas global stablecoins}

Aprovechando las asambleas de primavera y anuales del Fondo Monetario Internacional se suelen mantener reuniones de ministros de Finanzas y gobernadores de bancos centrales del G20. En el caso de las asambleas $\triangleright$ 
del pasado octubre de 2019 , lo más relevante de dicha reunión ministerial ha sido la emisión de sendos comunicados sobre fiscalidad internacional, con vistas a apoyar los trabajos en curso sobre fiscalidad digital y reiterando el compromiso de alcanzar un acuerdo final al respecto a finales de 2020, y sobre las mal denominadas global stablecoins ${ }^{1}$, para subrayar la necesidad de analizar los riesgos derivados de las innovaciones financieras y de resolverlos antes de que este tipo de iniciativas comiencen a operar.

\subsection{Fiscalidad internacional. Digitalización de la economía}

La transformación digital ofrece grandes oportunidades para la economía en términos de mejora de la productividad y eficiencia, pero también desafíos, en especial respecto a la eficacia y control de las políticas económicas.

Un ejemplo de esto último es el sistema fiscal internacional actual, cuyas normas no están adaptadas a los nuevos modelos de negocio. En concreto, se produce una desconexión entre la jurisdicción en la que se genera el valor y aquella en la que las empresas tributan, en la medida en que la digitalización permite a las empresas desarrollar sus actividades sin necesidad de contar con presencia física, así como deslocalizar activos intangibles a jurisdicciones de escasa o nula tributación.

Desde su elevación al foro de líderes en 2008, el G20 ha venido desempeñando un

1 Cabe definir las global stablecoins como criptomonedas vinculadas a un activo o cesta de activos con vistas a reducir su volatilidad. No obstante, dicho vínculo no garantiza necesariamente su estabilidad, de modo que su caracterización como stable resulta equívoca para los inversores y consumidores. Así, resulta preferible utilizar un término alternativo, como asset-backed tokens. papel clave en el impulso de la mejora de las normas fiscales internacionales a través del encargo de trabajos al respecto a la Organización para la Cooperación y el Desarrollo Económico (OCDE), bajo cuatro líneas de acción fundamentales: (i) refuerzo del cumplimiento de los estándares desarrollados por el denominado Foro Global sobre transparencia e intercambio de información por motivos fiscales; (ii) el desarrollo a través del conocido como Marco Inclusivo de medidas para evitar la elusión fiscal de empresas multinacionales a través del traslado de sus beneficios a países de nula o escasa tributación, fenómeno conocido como "erosión de la base imponible y traslado de beneficios» (Base Erosion and Profit Shifting, BEPS); (iii) análisis del impacto de la incertidumbre fiscal sobre el comercio y la inversión transfronterizos; y (iv) abordar los retos para la fiscalidad derivados de la digitalización de la economía.

Respecto a esta última línea de trabajo, el Marco Inclusivo presentó el año pasado un programa de trabajo que fue ratificado por los ministros de Finanzas y gobernadores de bancos centrales del G20 en junio de 2019 y por sus líderes en la cumbre del mismo mes y que incluye dos pilares:

- El primero gira en torno a las reglas de nexo y asignación de beneficios, tratando de determinar bajo qué circunstancias una jurisdicción tiene derecho a gravar los beneficios de una empresa no residente y la cuantía de beneficios que puede gravar.

- El segundo tiene como objetivo dar respuesta al problema de la deslocalización de las empresas a jurisdicciones con nula o muy baja tributación a través del establecimiento de un nivel mínimo $\triangleright$ 
de tributación global, permitiendo que las jurisdicciones puedan gravar la diferencia hasta ese nivel mínimo.

Aunque existe un amplio consenso acerca de la preferencia por alcanzar una solución a nivel internacional, algunos países han optado por aprobar medidas unilaterales para hacer frente al problema de la digitalización, con el compromiso de adaptarlas al acuerdo internacional cuando este se alcance. Así, resulta clave que en el seno del Marco Inclusivo de la OCDE se alcance un acuerdo, lo antes posible, para evitar una proliferación de medidas unilaterales que podrían dar lugar a problemas de tributación duplicada o inconsistente.

Con el objetivo de facilitar las negociaciones, el secretariado de la OCDE presentó a consulta pública en octubre de 2019 una «Propuesta Unificada» caracterizada por:

- Aplicarse a modelos de negocio dirigidos al consumidor y con un fuerte componente digital, excluyéndose las industrias extractivas.

- Incluir un nuevo criterio de sujeción que no está condicionado por la presencia física, sino que depende del nivel de ventas.

- Permitir la asignación de una parte del beneficio residual a una jurisdicción aunque la empresa no tenga una presencia física directa o indirecta a través de distribuidores no vinculados.

El próximo hito es la expectativa de que el Marco Inclusivo de la OCDE alcance, en enero de 2020, un acuerdo en torno a las líneas generales del diseño de ambos pilares y presente un informe con una propuesta final hacia finales de 2020.

\subsection{Las mal denominadas global stablecoins}

En la actualidad, más de 1.700 millones $^{2}$ de adultos siguen sin tener acceso a la banca tradicional. Sin embargo, dos tercios de ellos cuentan con un teléfono móvil, y un cuarto del total de los adultos sin acceso a una cuenta bancaria tiene acceso a internet. Además, el volumen de remesas a los países en desarrollo representa casi tres veces el de la ayuda oficial al desarrollo ${ }^{3}$, a pesar de su elevado coste de envío ${ }^{4}$. Como respuesta, en junio de 2019, Facebook informó sobre sus planes para crear una moneda digital basada en la tecnología blockchain y que contaría con el respaldo de una reserva de activos diseñada para aportarle valor intrínseco.

Este anuncio se produjo justo antes de la cumbre de líderes del G20 de Osaka, en cuyo comunicado se menciona de forma indirecta. En concreto, se señala: «Aunque los criptoactivos no representan por el momento un riesgo para la estabilidad financiera global, estamos siguiendo estrechamente su evolución y permanecemos alertas a sus riesgos existentes y emergentes». Por otro lado, se da la «bienvenida al trabajo en curso de la Junta de Estabilidad Financiera [Financial Stability Board, FSB] y de otros organismos fijadores de estándares» y se les solicita que "asesoren sobre respuestas multilaterales adicionales que resulten necesarias». Además, en el comunicado de prensa que se publicó durante las asambleas de octubre de 2019 se solicita al FMI que analice sus implicaciones macroeconómicas, $D$

\footnotetext{
2 Según datos para 2017 de la base Global Findex elaborada por el Banco Mundial.

3529.000 millones de dólares de remesas frente a 149.300 millones de dólares de ayuda oficial al desarrollo en 2018.

4 Según la base de datos de precios mundiales de remesas del Banco Mundial, el coste promedio de enviar 200 dólares fue de alrededor de un $7 \%$ en el primer trimestre de 2019.
} 
incluyendo asuntos relacionados con la soberanía monetaria.

A lo largo de 2020 se analizarán las recomendaciones de las diferentes organizaciones internacionales, en concreto las propuestas de política económica para reforzar el marco regulatorio.

\subsection{Otros temas que serán abordados por la presidencia saudí del G20 a lo largo de 2020}

La fiscalidad internacional y las mal denominadas global stablecoins serán, sin duda, los temas estrella durante la presidencia del G20 de Arabia Saudí en 2020, pero todas las presidencias, además de continuar con los trabajos existentes, introducen temas nuevos prioritarios para sus países y con los que pretenden dejar su impronta.

En el caso de la presidencia saudí, el tema transversal elegido es el de la igualdad de oportunidades, en especial para mujeres y jóvenes, teniendo previsto elaborar un documento de buenas prácticas con ejemplos de medidas nacionales de los países del G20. En este sentido, hay que tener en cuenta que Arabia Saudí es el país del G20 más joven por media de edad de su población y que desde el ascenso, en junio de 2017, de Mohammad bin Salman (nacido en 1985) al puesto de príncipe heredero se han adoptado en el país varias medidas favorables a mejorar la posición de la mujer en la sociedad saudí.

Adicionalmente, los saudíes han propuesto otras prioridades en el resto de líneas de trabajo habituales del G20. Una línea común a varias de ellas es la consideración de las nuevas tecnologías, como la inclusión financiera digital (centrándose específicamente en mujeres, jóvenes y pymes), la incorporación de las tecnologías a las infraestructuras, así como el análisis específico de los asuntos FinTech bajo cuatro líneas de trabajo (los pagos transfronterizos, la aplicación de las nuevas tecnologías a la regulación —-RegTech-y supervisión -SupTech—, el grado de preparación de los participantes del mercado para la transición del libor y las implicaciones de la entrada de grandes empresas tecnológicas -BigTechen los mercados financieros). En cuanto a los trabajos sobre arquitectura financiera internacional, los saudíes han fijado como prioridad el desarrollo de los mercados de capitales para reducir la dependencia de la deuda externa.

\section{Conclusiones}

Los ocho años de Christine Lagarde al mando del FMI han estado marcados por el impacto de la crisis financiera global de 20082009 y el estallido de la crisis de la zona euro en 2010-2011. Estos dos factores han hecho que la actividad de préstamo del fondo haya virado de las economías emergentes y en desarrollo hacia economías avanzadas, en las que se ha concentrado el porcentaje más elevado de las ayudas, destacando los programas de Grecia, Irlanda y Portugal. En este contexto tan complicado, Lagarde ha sido capaz de garantizar que el FMI siga siendo una institución relevante y además ha sabido adaptarla al nuevo contexto, dándole un mayor peso en la labor supervisora del Fondo a aspectos crecientemente importantes pero hasta ahora ignorados o infraconsiderados, como el cambio climático, la inclusión, la igualdad de género o la corrupción.

El pasado octubre, la primera mujer al mando del FMI dejó paso a otra mujer, la búlgara Kristalina Georgieva, en un contexto aparentemente menos complejo que el que tuvo que $\triangleright$ 
afrontar Lagarde en 2011, pero lleno de riesgos a la baja. Para afrontar estos, y ante la incertidumbre geopolítica y las amenazas al multilateralismo, en estas últimas asambleas se ha evidenciado la intención del FMI, haciendo uso de la representación casi universal que le otorgan sus 189 Estados miembros, de erigirse en adalid de la cooperación global para mantener la solidez del sistema financiero internacional.

\section{Bibliografía}

Banco Mundial (8 de abril de 2019). Record High Remittances Sent Globally in 2018. Recuperado de https://www.worldbank.org/en/news/pressrelease/2019/04/08/record-high-remittancessent-globally-in-2018

Comité Monetario y Financiero Internacional (19 de octubre de 2019). Comunicado de la Cuadragésima Reunión del CFMI. Recuperado de https:// www.imf.org/es/News/Articles/2019/10/19/ communique-of-the-fortieth-meeting-of-the-imfc

Fondo Monetario Internacional (2019). Fiscal Monitor: How to Mitigate Climate Change. Recuperado de https://www.imf.org/en/Publications/FM/lssues/2019/09/12/fiscal-monitor-october-2019

Fondo Monetario Internacional (2019). Global Financial Stability Report. Recuperado de https:// www.imf.org/en/Publications/GFSR/Issues/2019/ 10/01/global-financial-stability-report-october-2019

Fondo Monetario Internacional (2019). La Agenda Mundial de Políticas de la Directora Gerente: Actualización. Superar las diferencias mediante la cooperación multilateral. Recuperado de https://www.imf.org/en/Publications/Policy-Papers/Issues/2019/10/16/am2019-gpa-101719

Fondo Monetario Internacional (2019). World Economic Outlook. Recuperado de https://www.imf. org/en/Publications/WEO/Issues/2019/10/01/ world-economic-outlook-october-2019
G20 (8-9 de junio de 2019). Communiqué. G20 Finance Ministers and Central Bank Governors Meeting. Recuperado de https://www.mof.go.jp/ english/international_policy/convention/g20/ communique.htm

G20 (29 de junio de 2019). G20 Osaka Leaders' Declaration. Recuperado de http://www.g20.utoronto.ca/2019/2019-g20-osaka-leaders-declaration. html

G20 (18 de octubre de 2019). Press Release on Global Stablecoins. Recuperado de http://www.g20. utoronto.ca/2019/2019-g20-finance-stablecoins. html

G20 (18 de octubre de 2019). Press Release on International Taxation. Recuperado de http://www. g20.utoronto.ca/2019/2019-g20-finance-taxation. html

Grupo del Banco Mundial (2017). The Global Findex database 2017. Measuring Financial Inclusion and the Fintech Revolution. Recuperado de http://documents.worldbank.org/curated/ en/332881525873182837/pdf/126033-PUB-PUBLIC-pubdate-4-19-2018.pdf

Malpass, D. (27 de noviembre de 2017). Statement of Under Secretary David Malpass Before the U.S. Senate Foreign Relations Subcommittee on Multilateral International Development, Multilateral Institutions, and International Economic, Energy, and Environmental Policy. Recuperado de https:// home.treasury.gov/news/press-releases/sm555

OCDE (2019). El enfoque unificado: La propuesta del Secretariado relativa al Primer Pilar. Recuperado de https://www.oecd.org/tax/beps/documento-para-consulta-publica-el-enfoque-unificado-la-propuesta-del-secretariado-relativa-al-primer-pilar.pdf

OCDE (2019). Programme of Work to Develop a Consensus Solution to the Tax Challenges Arising from the Digitalisation of the Economy. Recuperado de https://www.oecd.org/tax/beps/ programme-of-work-to-develop-a-consensussolution-to-the-tax-challenges-arising-from-thedigitalisation-of-the-economy.pdf 